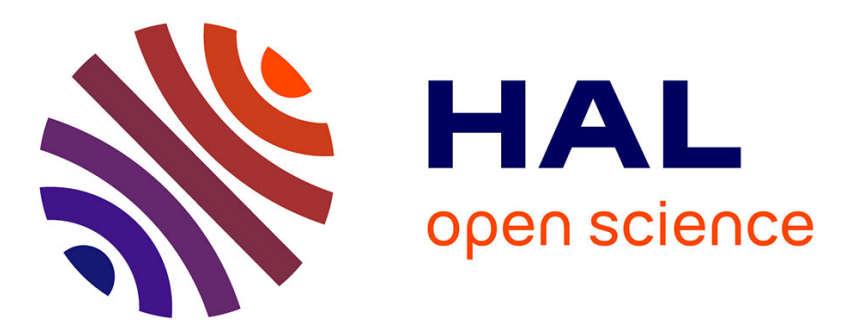

\title{
Analysis and possible estimation of keyhole depths evolution, using laser operating parameters and material properties
}

Rémy Fabbro, Morgan Dal, Patrice Peyre, Fredéric Coste, Matthieu Schneider, V Gunenthiram

\section{To cite this version:}

Rémy Fabbro, Morgan Dal, Patrice Peyre, Fredéric Coste, Matthieu Schneider, et al.. Analysis and possible estimation of keyhole depths evolution, using laser operating parameters and material properties. Journal of Laser Applications, 2018, 30, pp.Article number 032410. 10.2351/1.5040624 . hal-01824471

\section{HAL Id: hal-01824471 \\ https://hal.science/hal-01824471}

Submitted on 27 Jun 2018

HAL is a multi-disciplinary open access archive for the deposit and dissemination of scientific research documents, whether they are published or not. The documents may come from teaching and research institutions in France or abroad, or from public or private research centers.
L'archive ouverte pluridisciplinaire HAL, est destinée au dépôt et à la diffusion de documents scientifiques de niveau recherche, publiés ou non, émanant des établissements d'enseignement et de recherche français ou étrangers, des laboratoires publics ou privés. 


\title{
Analysis and possible estimation of keyhole depths evolution, using laser operating parameters and material properties
}

\author{
Remy Fabbro, Morgan Dal, Patrice Peyre, Frédéric Coste, Matthieu Schneider, and \\ Valerie Gunenthiram \\ PIMM Laboratory, Ensam-Cnrs-Cnam, 151, Bd. De l'Hôpital, 75013 Paris, France
}

\begin{abstract}
The authors propose an analysis of the effect of various operating parameters on the keyhole depth during laser welding. The authors have developed a model that uses the analysis of the thermal field obtained in $2 \mathrm{D}$ geometry, which is mainly defined by the characteristic Peclet number. This allows us to show that the dependence of the aspect ratio $R$ of the keyhole with the operating parameters of the process is a function of two parameters: a normalized aspect ratio $R_{0}$, controlled by the incident laser power and the spot diameter, and a characteristic speed $V_{0}$ related to the process of heat diffusion. The resulting general law $R=f\left(R_{0}, V / V_{0}\right)$ appears to be very well verified by different experimental data and allows to define mean thermophysical parameters of the used materials. These data can then be used for keyhole depths prediction for any subsequent operating parameters of the same material. This model also allows us to define precisely a criterion for a keyhole threshold generation. The authors will apply the derived procedure to successfully analyze experiments on materials with very different thermophysical properties (such as steel alloys and copper), with various focal spots, incident laser powers, and welding speeds.
\end{abstract}

Key words: laser welding, keyhole, keyhole depth estimation

\section{INTRODUCTION}

Laser beam welding in deep penetration mode, using a keyhole $(\mathrm{KH})$ mode, is now a very attractive and popular process. Because of the possible wide range of laser operating parameters, $\mathrm{KH}$ depths ranging from a few hundred microns to several tens of millimeters can be easily produced in many different metallic materials. However, the predictability of these $\mathrm{KH}$ depths as a function of the operating parameters and the thermophysical properties of the used material is very far from being achieved. Specific experiments producing tables seem to be up to now the best way for obtaining reliable data. However, since almost 40 years, in connection with the growing knowledge of physical phenomena occurring during this type of interaction, many more or less complex models have been developed with variable satisfactory results. Following the pioneering work of Rosenthal, ${ }^{1}$ many studies analyzed the thermal field induced by a line source ${ }^{2}$ or a cylindrical KH (Refs. 3-7) moving inside the workpiece. Thereafter, numerical simulations were used and due to the increased computational capability and the improvement of numerical algorithms, much more physical processes could be added to achieve 3D complex numerical simulations describing the melt pool hydrodynamics, and taking into account free surfaces deformation, vapor and plasma effects, or multiple beam reflections inside the $\mathrm{KH} .{ }^{8-14}$ However, it is clear that these complex numerical simulations can only be handled by skilled researchers and moreover, they are not practically realized in realistic frame time. If we are only interested in estimating the $\mathrm{KH}$ depth, with a satisfying precision for a given set of operating parameters and materials, we need a much simpler method. By using an intermediate approach, initially proposed by Lankalapalli et al., ${ }^{6}$ where a thermal analysis of this process coupled to the results of $2 \mathrm{D}$ simulations is used, we will show how this approach can be adapted to the analysis of different experimental results and then for estimating the $\mathrm{KH}$ depths for subsequent operating parameters.

In the first part of this paper, we will define a criterion for $\mathrm{KH}$ generation. Then, after the description of the thermal model and its improvement, we will discuss how it can be adapted to the KH depth analysis. Finally, we will discuss its application to several experimental situations concerning the welding of different materials.

\section{THRESHOLD FOR KH GENERATION}

During a laser welding process, a $\mathrm{KH}$ appears only if specific conditions on operating parameters are met. Figure 1 shows the transition of the melt pool to a $\mathrm{KH}$ geometry, when for example one varies the welding speed $V$, for a given incident power $P$. At high welding speed $V$, a melt pool appears when the surface temperature reaches the melting temperature $T_{m}$ [Fig. 1(a)]. Then if the welding speed is decreased, the surface temperature reaches the evaporation one $T_{v}$, and the surface below the laser spot begins to be depressed by the recoil pressure [Fig. 1(b)], but the reflected incident beam is still reflected upwards, which carries away about typically $60 \%$ from the incident laser power (at $1.06 \mu \mathrm{m}$, on steel alloys ${ }^{15}$ ). By a further reduction of the welding speed, due to the resulting increase in surface 


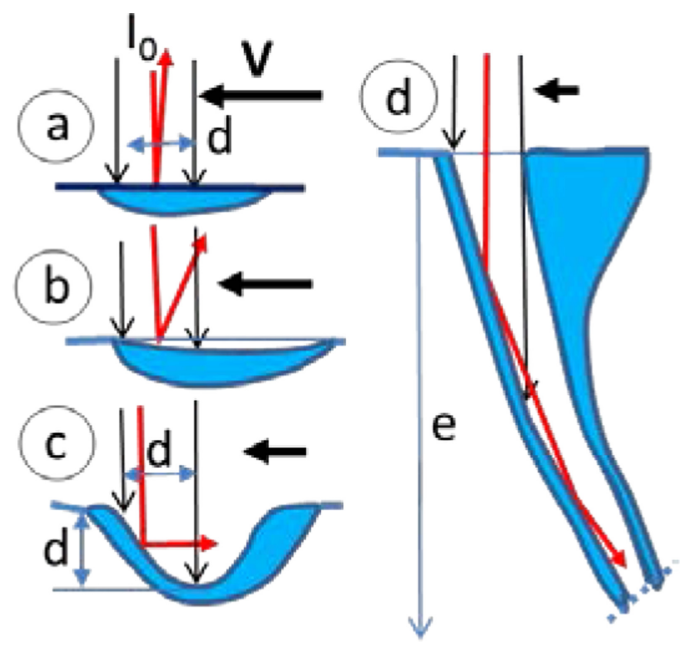

FIG. 1. (a-d) As a function of the welding speed $V$, evolution of the melt pool geometry leading to the $\mathrm{KH}$ formation.

temperature and the subsequent recoil pressure, the deformation of the melt pool surface increases and the inclination of what will be the $\mathrm{KH}$ front can reach $45^{\circ}$ [Fig. 1(c)]; the reflected beam is then horizontal, and of course, any further reduction of the welding speed will make it to be directed downwards [Fig. 1(d)], adding its contribution to an increased penetration leading to a $\mathrm{KH}$, where quite all the incident beam is trapped. Following this scheme, it is clear that the threshold for $\mathrm{KH}$ generation is defined by finding the conditions where the inclination angle of the $\mathrm{KH}$ front is about $45^{\circ}$, or equivalently, when the penetration depth $e$ is equal to the laser spot diameter $d: e \approx d$.

For finding these conditions, we use a previous model that gives the $\mathrm{KH}$ front inclination angle and the penetration depth $e$ resulting of the first impact of the laser beam. ${ }^{16}$ The corresponding penetration depth $e$ is then given by

$$
\frac{e}{d} \approx \frac{4 A_{0}}{\pi H_{0}} \frac{P}{V \cdot d^{2}}
$$

where $A_{0}$ is the absorption coefficient under normal incidence, $P$ is the incident power $(\mathrm{W})$, and $H_{0}\left(\right.$ in $\mathrm{J} / \mathrm{m}^{3}$ ) is the enthalpy necessary to the melting of the incoming solid. By using the "piston model," 17 it is easy to show that for operating conditions where the evaporation process is negligible, $H_{0}=h_{m}+h_{s} \cdot f(P e)$, where $h_{m}=\rho_{s}\left[C_{p s}\left(T_{m}-T_{0}\right)+L_{m}+C_{p m}\left(T_{v}\right.\right.$ $\left.-T_{m}\right) / 2$ ] is a modified enthalpy at fusion [an average temperature $\left(T_{m}+T_{v}\right) / 2$ of the sideways ejected liquid is considered], and $h_{s}=\rho_{s} C_{p s}\left(T_{m}-T_{0}\right)$ is the enthalpy at melting. The function $f(P e)=u P e^{-v}$ defines the conduction losses, where $P e=V \cdot d / 2 k$ is the usual Peclet number ( $k$ : heat diffusivity), and the constants $u$ and $v$ depend of the considered geometry [for example, $u \approx 2^{0.7}$ and $v=0.7$, for a strip of width $d$ (Ref. 18)].

With the condition $e=d$ in Eq. (1), it is then possible to define the threshold for $\mathrm{KH}$ formation which becomes:

$$
\frac{P}{V \cdot d^{2}}=\frac{\pi}{4} \frac{H_{0}}{A_{0}}
$$

It is worth noticing that Eq. (2) can be rewritten as $A_{0} \cdot P=\left(\pi \cdot d^{2} / 4\right) \cdot V \cdot H_{0}$, which appears to be an energy balance equation defining the absorbed power $A_{0} \cdot P$ required for delivering the heat enthalpy $H_{0}$, to a volume rate $\left(\pi \cdot d^{2} / 4\right) \cdot V$ of solid material $\left(\pi \cdot d^{2} / 4 \approx \mathrm{d}^{2}\right.$ is the section of the incoming solid material) [see Fig. 1(c)]. The experimental validation of this threshold determination will be discussed at the end of Sec. III.

\section{ESTIMATION OF THE KH DEPTH}

The KH geometry used here is shown in Fig. 2. We assume a vertical cylindrical KH geometry, with its surface at the evaporation temperature $T_{v}$ of the material and all the incident laser power $P$ is homogenously absorbed on the $\mathrm{KH}$ wall surface, along the $\mathrm{KH}$ depth $e$. Therefore, $e$ verifies the relation $e=P / P_{z}$, where $P_{z}=d P / d z$ is the absorbed power per unit depth injected inside the $\mathrm{KH}$ necessary for maintaining the $\mathrm{KH}$ surface at $T_{v}$.

$P_{z}$ can be determined by analyzing the resulting 2D-thermal field for this 2D geometry. If we assume constant thermophysical properties, no solid-liquid phase change, and a welding speed $V$ along the $x$-direction, the 2D heat equation is then: ${ }^{1}$

$$
\rho C_{p} \frac{\partial T}{\partial t}+\rho C_{p} V \frac{\partial T}{\partial x}=K\left(\frac{\partial^{2} T}{\partial x^{2}}+\frac{\partial^{2} T}{\partial y^{2}}\right)
$$

with $T=T_{0}$ at $x, y \rightarrow \pm \infty$, and $T=T_{v}$ for $r=\left(x^{2}+y^{2}\right)^{1 / 2}=d / 2$. ( $K$ : heat conductivity; $C_{p}$ : heat capacity).

If one introduces the dimensionless variables:

$T^{\prime}=T /\left(T_{v}-T_{0}\right), x^{\prime}=2 \cdot x / d, y^{\prime}=2 \cdot y / d, r^{\prime}=2 \cdot r / d$ and $t^{\prime}=t / \tau$ (with $\tau=d^{2} / 4 k$ ), then Eq. (3) becomes:

$$
\frac{\partial T^{\prime}}{\partial t}+P e \cdot \frac{\partial T^{\prime}}{\partial x^{\prime}}=\frac{\partial^{2} T^{\prime}}{\partial x^{\prime 2}}+\frac{\partial^{2} T^{\prime}}{\partial y^{\prime 2}} .
$$

Equation (4) shows that the Peclet number $P e$ is the only parameter controlling the thermal field and its resulting gradient at the KH surface. Using Finite Element Modeling (FEM), it is easy to check that the stationary state is obtained for

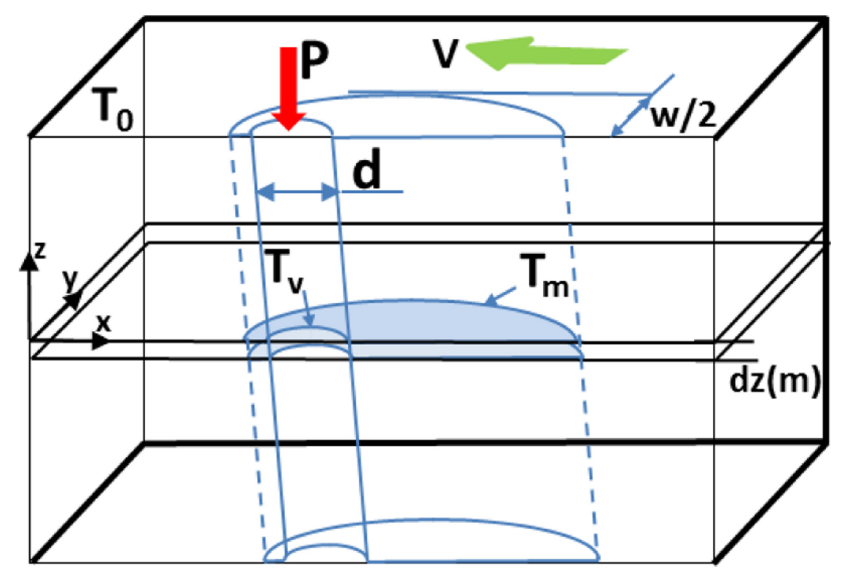

FIG. 2. Sketch of the longitudinal section of an inclined keyhole. The incident beam power is $P$. The KH surface is $T_{v}$ and its diameter is $d$. The width of the melting isotherm $T_{m}$ is $w$. Welding speed is $V$. 
$t^{\prime} \geq 3-4$. An analytical solution of this thermal field obtained in stationary conditions has already been derived using the polar coordinates $(r, \theta) .^{1,4-6,19}$ It can also be determined from 2D FEM calculation. We did here because it also allows us to consider the input laser power devoted to the solid-liquid phase change, which cannot be derived from the analytical solution of Eq. (4).

From the resulting thermal field, for stationary conditions, it is then possible to determine the input power $P_{z}$ conducted through the $\mathrm{KH}$ surface:

$$
\begin{aligned}
P_{z} & =\int_{0}^{2 \pi}-K\left(\frac{\partial T(r, \theta)}{\partial r}\right)_{r=d / 2} r d \theta \\
& =K\left(T_{v}-T_{0}\right) \cdot \int_{0}^{2 \pi}-K\left(\frac{\partial T^{\prime}\left(r^{\prime}, \theta\right)}{\partial r^{\prime}}\right)_{r^{\prime}=1} d \theta \\
& =K\left(T_{v}-T_{0}\right) \cdot g(P e) .
\end{aligned}
$$

The function $g(\mathrm{Pe})$ has been plotted in Fig. 3. Equation (5) shows that as the welding speed (or the Peclet number) increases, the input power $P_{z}$ at the KH surface, which is also dissipated from this zone by convection, must increase in order to keep a constant surface temperature of the $\mathrm{KH}$ at $T_{v}$.

Lankalapalli et al. ${ }^{6}$ have shown that $g(P e)$, on the range $0<P e<5$, could be approximated by an empirical equation expressed by a third degree polynomial expression. But in the present work, for the analysis of experimental results, we will see below the great interest of a very different approach: One uses a linear relation $g(P e)=m \cdot P e+n$ instead of the third degree polynomial expression.

But because of the non-linear behavior of $g(\mathrm{Pe})$, and in order to keep a good accuracy on $g(P e)$ whatever the value of $P e$ (i.e., with a correlation index greater than 0.99 ), one must therefore use different linear relations defined by the pair $(m, n)$, along the $P e$ range. This means that the parameters $m$ and $n$ must vary with $P e$. For $0.01 \leq P e \leq 10$, we have defined five ranges of $\mathrm{Pe}$, each having a variation dynamics of $\mathrm{Pe}$ of about 6 , and for which the average value

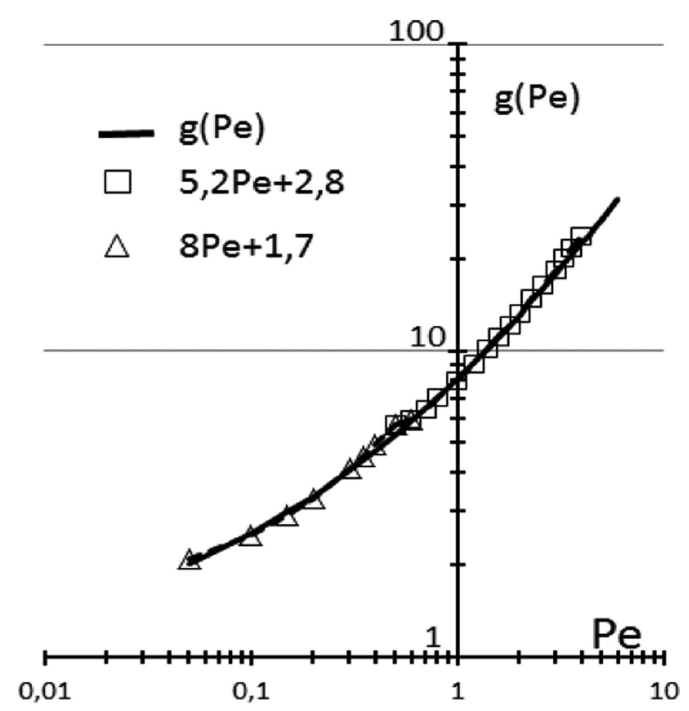

FIG. 3. Variation $g(P e)$ as a function of the Peclet number Pe. Examples of two linear fits are shown. of the pair $(m, n)$ has been determined. The values of the pairs $(m, n)$ on these five different ranges have been reported in Fig. 4. It is worth recalling that most of the experiments usually realized on steels have corresponding Peclet numbers in the range $0.2<P e<6$, where typically $m \approx 5$ and $n \approx 3$. Two examples of these linear fits have been also reported in Fig. 3.

We have now all the elements for determining the aspect ratio $R=e / d$. Using Eq. (5), one can write:

$$
\begin{aligned}
R & =\frac{e}{d}=\frac{P}{d \cdot P z}=\frac{P}{d \cdot K \cdot\left(T_{v}-T_{0}\right) \cdot g(P e)} \\
& =\frac{P}{d \cdot K \cdot\left(T_{v}-T_{0}\right) \cdot(m \cdot P e+n)} .
\end{aligned}
$$

As $P e=V \cdot d / 2 \cdot \kappa$, Eq. (6) can be rewritten in a muchgeneralized form:

$$
R=\frac{R_{0}}{\left(1+V / V_{0}\right)}
$$

with

$$
R_{0}=\frac{P}{n \cdot d \cdot K \cdot\left(T_{v}-T_{0}\right)} \text { and } V_{0}=2 \frac{n}{m} \frac{k}{d}
$$

The interest of the very simple Eq. (7a) is that it gives the scaling law of the $\mathrm{KH}$ depth with all the parameters of this problem. Moreover, it reproduces the variations of the $\mathrm{KH}$ depth $e$ that are usually observed experimentally: at high welding speeds (if $V \gg V_{0}, V_{0}$ being characteristic of some "speed" of heat diffusion), one finds $R \approx R_{0} \cdot V_{0} / V$, and it is known that at high welding speeds, the $\mathrm{KH}$ depth $e$ is roughly inversely proportional to the welding speed $V$. On the contrary, when the welding speed decreases, (or $V<V_{0}$ ), it is observed that the penetration depth $e$ increases and then saturates. Equation (7) reproduces this behavior and the maximum aspect ratio is then equal to $R_{0}$. These trends can

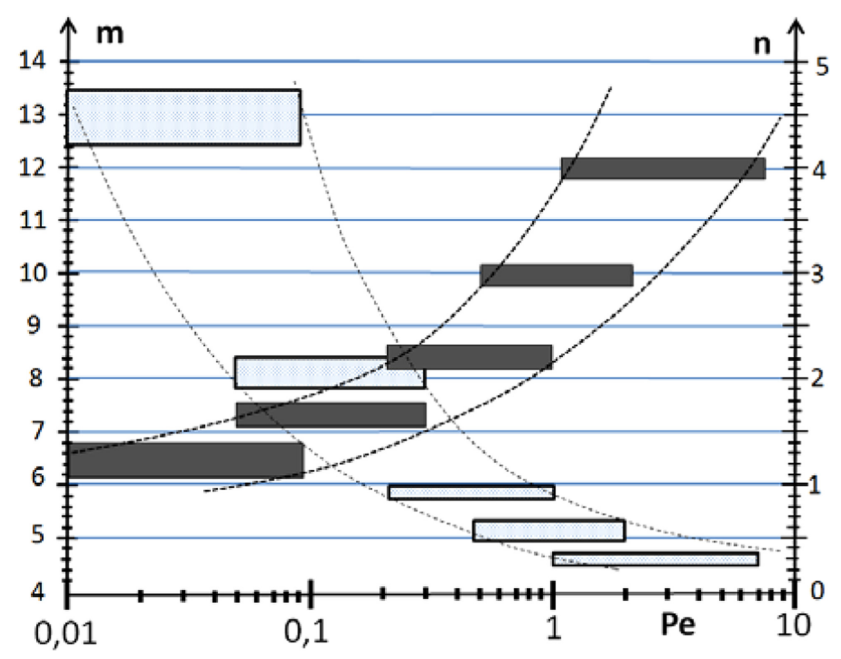

FIG. 4. Inside five selected $P e$ intervals, ranging from $P e=0.01$ to $P e=10$, mean values of the pair of parameters $m$ (empty symbols) and $n$ (filled symbols) used for defining the corresponding best fit for a linear variation of $g(P e)$, as $g(P e)=m P e+n$. 
be understood by considering the ratio convection/conduction losses that are of course directly related to the Peclet number: at low $\mathrm{Pe}$ (or low $V$ ), convection becomes negligible (and even null for $V=0$ ); the input power $P_{z}$ inside the $\mathrm{KH}$ is small and so the incident laser power $P$ can be distributed on a greater (and maximum) depth. At high $P e$ (or high $V$ ), convection dominates and makes $P_{z}$ to increase quite linearly with the welding speed: the penetration depth then decreases.

Remark 1: From Eq. (7), one sees that the aspect ratio $R$ depends on two operating parameters: $P / d$ and $V$. But Eq. (7a) can also be rewritten as

$$
R=\frac{P / V d^{2}}{\Delta H_{v}} \cdot \frac{1}{\left(1+V_{0} / V\right)},
$$

with

$$
\Delta H_{v}=0.5 m \rho C_{p}\left(T_{v}-T_{0}\right) .
$$

In that case, one can see that $R$ is mainly defined by the ratio of two volumetric energy densities (in $\mathrm{J} / \mathrm{m}^{3}$ ): $P / V d^{2}$ is related to the operating parameters, and $\Delta H_{v}$ to the thermophysical properties of the material, which is a kind of enthalpy parameter that contains the evaporation temperature $T_{v}$. Moreover, using Eq. (8), one can find that the threshold for $\mathrm{KH}$ generation, i.e.: $R \geq 1$, is defined by

$$
\frac{P}{V d^{2}} \geq \frac{\Delta H_{v}}{A_{0}} .
$$

In Eq. (9), when $R=1$, we must take into account the absorptivity of the incident beam for these conditions, which is near $A_{0}$, and not quite $100 \%$ as in the case of deep penetration when a large beam trapping occurs. More rigorously, in Eq. (7) or (8), one should also take into account the level of absorptivity of the incident laser beam, which is defined by the number of multiple reflections inside the KH that depends on the aspect ratio $R$. It is known that this absorptivity increases from $A_{0}$ to nearly $100 \%$ when the aspect ratio $R$ increases from 0 to about $8-10$, due to increased beam trapping efficiency. ${ }^{20,21}$ It is also interesting to notice that the two $\mathrm{KH}$ thresholds defined by Eqs. (9) and (2) have the same order of magnitude, although obtained with very different approaches [see Eqs. (2) and (9)]. Indeed, it is found that $\Delta H_{v} / H_{0} \approx 3$ (thermophysical parameters of SS304 L were used).

Remark 2: Equation (8) can also be rewritten as an energy balance equation: $A_{0} \cdot P=V \cdot(e \cdot d) \cdot \Delta H_{v} \cdot\left(1+V_{0} / V\right)$ that gives a more physical interpretation of the obtained law. At high welding speed, $A_{0} \cdot P$ is the absorbed power necessary to give to the volume rate $V \cdot(e \cdot d)$ of solid material $(e \cdot d$ being the section of incoming solid material), the enthalpy $\Delta H_{v}$ that contains information on both the heating from $T_{0}$ to $T_{\mathrm{v}}$ and the overall heat diffusion process through the parameter $m$.

Remark 3: The scaling of the KH depths [Eq. (7)] with the thermophysical parameters shows that the $\mathrm{KH}$ depth increases with reduced heat conductivities $K$ or with low evaporation temperatures $T_{v}$. This is in agreement with the observed increase in the $\mathrm{KH}$ depth due to the decrease in $T_{v}$ as a result, for example, of welding under reduced ambient pressure. ${ }^{22}$

\section{EFFECT OF SOLID-LIQUID PHASE CHANGE}

The previous analysis has determined the heat input inside the $\mathrm{KH}$ without considering any solid-liquid phase change that generates the observed liquid melt pool during laser welding. It is therefore important to estimate the power that is necessary to add to the incident one previously determined, for this melt pool generation. In Fig. 2, one can see that the only part of the solid material that undergoes this phase change is located between $y= \pm w / 2$ ( $w$ being the width of the isotherm at $T_{m}$ ). As this part already undergoes the heating from $T_{0}$ to $T_{m}$, its subsequent melting requires only the enthalpy of fusion $L_{m}$. Therefore, as the material is flowing at the welding speed $V$, the excess of incident power (per unit depth) $\Delta P_{z m}$ necessary for this process is given by

$$
\Delta P_{z}^{m}=V w \rho L_{m}
$$

For evaluating $\Delta P_{z}^{m}$, the width $w$ of the melting isotherm must be known. Beck ${ }^{21}$ has proposed an analytical approximation for $w / d$ :

$$
w / d=1+G\left(T_{v}, T_{m}, T_{0}\right) P e^{-0.5},
$$

where

$$
G\left(T_{v}, T_{m}, T_{0}\right)=2^{3.5}\left(\frac{T_{v}-T_{m}}{T_{v}+T_{m}-2 T_{0}}\right)^{2}
$$

By using the FEM COMSOL software, ${ }^{23}$ we have verified the validity of Eq. (11), for the Peclet range $0.08<P e<10$. In order to quantify the importance of this excess of power $\Delta P_{z m}$, we have to compare it with the previously determined input power $P_{z}$ :

$$
\begin{aligned}
\Delta P_{z}^{m} & =\frac{V d \rho L_{m}(w / d)}{K\left(T_{v}-T_{0}\right)(m \operatorname{Pe}+n)} \\
& =2 \frac{L_{m}}{C_{p}\left(T_{v}-T_{0}\right)}\left(\frac{\operatorname{Pe}\left(1+G P e^{-0.5}\right)}{m \operatorname{Pe}+n}\right) .
\end{aligned}
$$

As typically $2 \cdot L_{m} /\left(C_{p} \cdot\left(T_{v}-T_{0}\right)\right) \approx 0.40$, it can be seen that the ratio $\Delta P_{z m} / P_{z}$ varies from 0 , at low $P e$ (or low welding speeds), to $\approx 0.40 / m \approx 8.5 \%$ at high welding speeds. Therefore, the power required to achieve this phase change is less than $10 \%$ of the input power used in the generation of the entire thermal field inside the workpiece. So, we will neglect this effect for the analysis of the experimental results described in Sec. V.

\section{APPLICATION TO THE ANALYSIS OF EXPERIMENTS}

The expression of the $\mathrm{KH}$ aspect ratio $R$ according to Eq. (7a) is very interesting, because it shows that $1 / R$ becomes a linear function of the welding speed $V$. Indeed, we see that

$$
\frac{1}{R}=\frac{1}{R_{0}}\left(1+\frac{V}{V_{0}}\right)=\frac{V}{R_{0} V_{0}}+\frac{1}{R_{0}}=a V+b .
$$


So, for a given set of experimental results, if one plots $1 / R$ as a function of the welding speed $V$, one should find a linear function whose slope is $a$ and the ordinate at the origin, $b$. Knowing $a$ and $b$, from Eq. (14), one can then determine easily $R_{0}$ and $V_{0}$ from:

$$
R_{0}=\frac{1}{b} \quad \text { and } \quad V_{0}=\frac{b}{a}
$$

It is this procedure that we have applied below to several experiments realized with different operating parameters or materials. The interest of this method is that it uses a fit of experimental data to define the parameters $R_{0}$ and $V_{0}$, and not Eq. (7b), which requires the knowledge of average values of $K$ and $k$, which are always difficult to define because of their strong dependence with temperature. Once $R_{0}$ and $V_{0}$ are estimated with this procedure, for example, for a given set of initial experiments, one can use them for predicting the $\mathrm{KH}$ depths for other operating parameters, for the same material.

Of course, by using directly Eqs. (7a) and (7b) with some "assumed" parameters for $K$ and $k$, one may have some initial estimate of the KH depths, which may be very useful for preliminary experiments.

\section{A. Analysis of several experiments}

We first applied here our procedure for the analysis of recently published data on $\mathrm{KH}$ depths obtained on St35 steel at $1.06 \mu \mathrm{m}$ laser wavelength for different incident laser powers and focal spots. ${ }^{24}$

In Fig. 5, the inverse of the aspect ratio $1 / R$ has been plotted, as a function of the welding speed, for the three incident laser powers: 2,5 , and $8 \mathrm{~kW}$, and two focal spot diameters: 0.78 and $0.38 \mathrm{~mm}$, used by Suder and Williams. ${ }^{24}$ The corresponding best linear fits for these data are also shown.

As in Fig. 5, we have plotted in Fig. 6 the variation of $1 / R$ as a function of welding speed, for different laser powers and focal spot diameters when laser welding of copper in deep penetration mode, from experiments of Heider et al. ${ }^{25}$

Due to the rather important uncertainties for determining $\mathrm{KH}$ depths at high welding speeds from the data collected

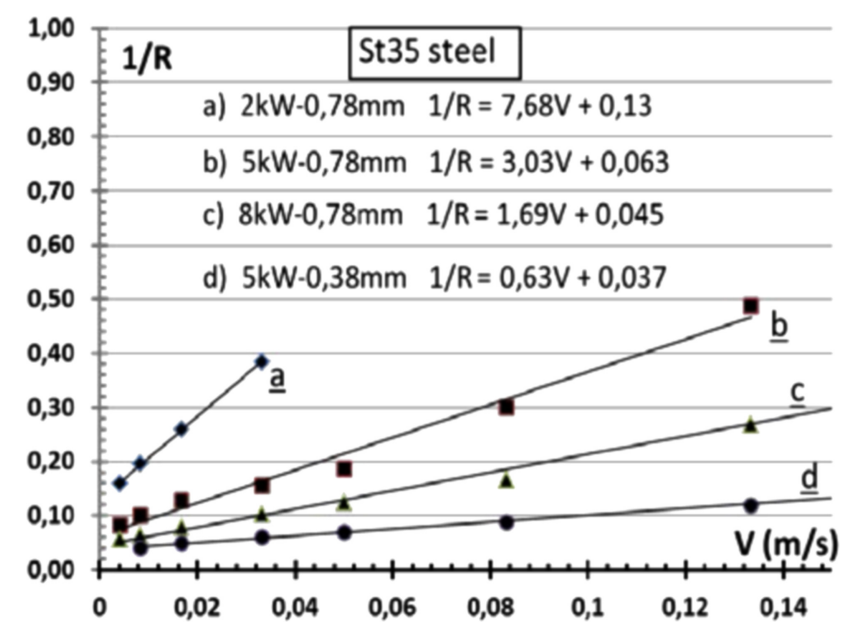

FIG. 5. For St35 steel and different operating parameters, plot of $1 / R$ as a function of the welding speed $V$ and corresponding best linear fits.

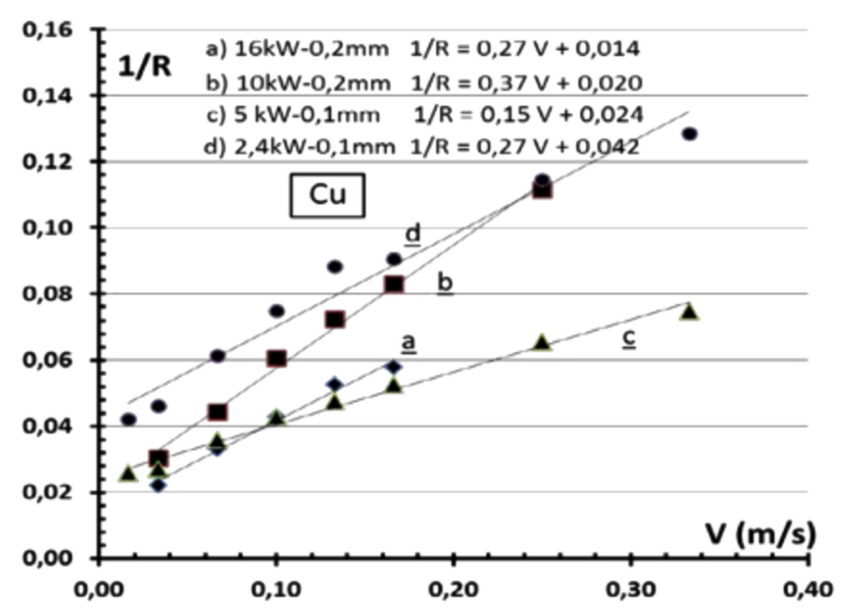

FIG. 6. For $\mathrm{Cu}$ and different operating parameters, plot of $1 / R$ as a function of the welding speed $V$ and corresponding best linear fits.

from Refs. 24, 25, only data for $R \geq 3$ have been reported in Figs. 5 and 6. Moreover, a high aspect ratio also should ensure a large trapping of the incident laser beam inside the $\mathrm{KH}^{20,21}$ and so less uncertainty on the beam absorptivity, which is quite $100 \%$, as well as a better 2D induced thermal field, which is one of the main constraints of our thermal model. For all these different experiments, one must notice the remarkable linear dependence of $1 / R$ with the welding speed $V$.

\section{B. Discussion}

From the slope $a$, and the ordinate at origin $b$ for each linear fit, and then using Eq. (15), the parameters $R_{0}$ and $V_{0}$ entering in Eq. (7a) have been determined.

For both materials, their values have been reported in Table I (with the label "fit"), and can be compared with those expected when Eq. (7b) is estimated (label "direct") by using mean thermophysical data at melting temperature ${ }^{26}$ and the $m$ and $n$ parameters derived from Fig. 4 on the corresponding $P e$ range of these experiments.

In Table I, the parameters $V_{0}$ and $R_{0}$ derived from the linear fits and those estimated from assumed mean values of $K$ and $k$ are rather close and similarly follow the expected tendencies: $R_{0 F}$ increases with the incident laser power and with a decrease in the focal spot diameter. For the St35 steel, the mean "diffusion" velocity $V_{0 F}$ of about $0.022 \mathrm{~m} / \mathrm{s}$ for the $0.78 \mathrm{~mm}$ experiments is doubled when the spot diameter decreases to $0.38 \mathrm{~mm}$. From the $V_{0 F}$ variations, on $\mathrm{Cu}$ experiments compared to the steel experiments, one can also see that our model catches fairly well the great difference of heat diffusivity and spot sizes between these two materials.

However, one can see that there are some differences between the "fitted" and "direct" parameters $R_{0}$ and $V_{0}$. This results mainly from the choice of the selected parameters $K$ and $k$ that are rather arbitrary, and also, in the case of the parameter $R_{O D}$, from some possible overestimation of the absorbed power $P$. One knows that absorptions of about $80 \%$ of the incident laser power are usually considered, even inside large aspect ratio KH. In Table I, one can remark that the $R_{0 D}$ is always greater than the "fitted" one. By applying some reduction of about $80 \%$ to the incident power, both values 
TABLE I. Parameters $V_{0}$ and $R_{0}$ from analysis of experiments (Refs. 24, 25).

\begin{tabular}{|c|c|c|c|c|c|c|c|c|}
\hline \multirow[b]{2}{*}{ Incident laser power $(\mathrm{kW})$} & \multicolumn{4}{|c|}{ Material: St35 steel } & \multicolumn{4}{|c|}{ Material: $\mathrm{Cu}$} \\
\hline & 2 & 5 & 8 & 5 & 16 & 10 & 5 & 2.4 \\
\hline Spot diameter $(\mathrm{mm})$ & 0.78 & 0.78 & 0.78 & 0.38 & 0.2 & 0.2 & 0.1 & 0.1 \\
\hline$V_{O F}(\mathrm{~m} / \mathrm{s})($ from fit $)$ & 0.017 & 0.021 & 0.027 & 0.059 & 0.05 & 0.06 & 0.16 & 0.15 \\
\hline$V_{0 D}(\mathrm{~m} / \mathrm{s})($ direct $)$ & 0.013 & 0.013 & 0.013 & 0.028 & 0.086 & 0.086 & 0.17 & 0.17 \\
\hline$R_{0 F}$ (from fit) & 7.7 & 15.9 & 22.2 & 27.0 & 69.4 & 48.8 & 40.5 & 23.5 \\
\hline$R_{0 D}($ direct $)$ & 7.6 & 19.0 & 30.5 & 39.1 & 84.1 & 52.6 & 52.6 & 25.2 \\
\hline Used parameters & \multicolumn{4}{|c|}{$\begin{array}{l}\left.K \approx 30 \mathrm{~W} / \mathrm{m} \cdot \mathrm{K}, k \approx 6 \cdot 10^{-5} \mathrm{~m}^{2} / \mathrm{s} \text { (Ref. } 26\right) \text { as } 0.15 \leq \\
P e \leq 5, m \approx 4.5 \text { and } n \approx 4\end{array}$} & \multicolumn{4}{|c|}{$\begin{array}{l}\left.K \approx 250 \mathrm{~W} / \mathrm{m} \cdot \mathrm{K}, k \approx 6 \cdot 10^{-5} \mathrm{~m}^{2} / \mathrm{s} \text { (ref. } 26\right) \text { as } 0.02 \leq \\
P e \leq 0.2, m \approx 10 \text { and } n \approx 1.5\end{array}$} \\
\hline
\end{tabular}

would be much closer. Furthermore, once the $P e$ range is given, the parameters $m$ and $n$ are well enough defined (from Fig. 4); so, one then could estimate mean values of $K$ and $k$ from the $R_{0 F}$ and $V_{O F}$ parameters. In doing so to the present results, one finds that the resulting mean values of $K$ and $k$ are slightly different from the assumed ones and they can be considered to be more representative of the mean thermophysical properties of this material for these welding conditions.

\section{KH depth estimation procedure}

The confidence resulting from the previous analysis allows us to propose a rather simple methodology for a rapid and easy $\mathrm{KH}$ depth estimation that can be described by the following steps:

- For a given material (that defines $T_{v}$, and mean values of $K$ and $k$ defined at fusion temperature of this material) and the set of operating parameters (incident laser power $P$, range of welding speeds $V$, spot diameter $d$ ), one first estimates the corresponding $\mathrm{Pe}$ range and therefore the corresponding parameters $m$ and $n$ to be used, from Fig. 4 for this $P e$ range.

- From these data, the parameters $R_{0 D}$ and $V_{0 D}$, from Eq. (7b), and finally the variation of the aspect ratio $R$ (or the $\mathrm{KH}$ depth) can be computed on this welding speed range [from Eq. (7a)].

However, if some experiments can be realized with these conditions (typically three different welding speeds are enough), the corresponding parameters $R_{0 F}$ and $V_{0 F}$ can be determined from the resulting linear plot of $1 / R$ with the welding speed $V$. For other operating parameters on the same material, $R_{0}$ and $V_{0}$ will then be easily extrapolated from the previously determined $R_{O F}$ and $V_{O F}$, by using their scaling laws from Eq. (7b) [where $V_{0 D} \propto d^{-1}$ and $R_{0 D} \propto(P / d)$ ].

This procedure can be tested with some results from Table I. Let us suppose that we have made some trials on St35 steel, at $5 \mathrm{~kW}$ with the $0.78 \mathrm{~mm}$ focal spot. From Table I, one sees that one would obtain $R_{0} \approx 15.9$ and $V_{0} \approx$ $0.013 \mathrm{~m} / \mathrm{s}$. If one uses these data for estimating $R_{0}$ for experiments that would be realized at $8 \mathrm{~kW}$ with the same focal spot, one finds that $R_{0} \approx 15.9(8 / 5)=25.4$, which is about $13 \%$ greater than the experimental value of 22.2 given in Table I for these operating conditions. For experiments that would be realized at $5 \mathrm{~kW}$ with a $0.38 \mathrm{~mm}$ focal spot, one would obtain: $R_{0} \approx 15.9(0.78 / 0.38)=32.6$, which is about
$17 \%$ greater than the corresponding experimental value of 27. This procedure can be applied to other operating conditions used in Table I and one finds that maximum typical differences of $20 \%$ can be seen between estimated and corresponding experimental values for $R_{0}$.

So ultimately, one can see that the observed linear dependence of $1 / R$ with the welding speed is very fruitful. One can add that this linear scaling can be observed in quite all the experiments dealing with laser welding in $\mathrm{KH}$ mode with high aspect ratios.

\section{CONCLUSION}

We have extended the Lankalapalli et al. ${ }^{6}$ approach in order to define a model that contains more physical insights and an easier analysis of the relevant parameters from experimental data. This rather simple model allows a rapid and easy estimation of the $\mathrm{KH}$ depth as a function of the operating parameters (incident laser power, focal spot diameter, welding speed) and thermophysical parameters of the used material, for experiments performed at $1.06 \mu \mathrm{m}$ laser wavelength. Based on the assumption of a homogeneous deposition of incident laser power along the walls of a vertical cylindrical $\mathrm{KH}$ at evaporation temperature, this model gives the resulting final aspect ratio $R$ as a function of the welding speed $V, R=R_{0} /\left(1+V / V_{0}\right)$, which depends on two parameters: $R_{0}$ represents the maximum aspect ratio obtained when the welding speed $V$ is zero, and a welding speed $V_{0}$ characteristic of the transition from conduction to convection dominated losses as the welding speed increases.

As this model shows that $R^{-1}$ is a linear function of the welding speed $V$, which is consistent with the experiments, both parameters $R_{0}$ and $V_{0}$ can then be easily determined from few experiments. Once $R_{0}$ and $V_{0}$ are determined for specific operating parameters, they can be adapted for any other operating conditions of incident laser power, focal spot or welding speed for the same material. Of course, one can use this model in a direct approach by assuming "mean" thermophysical parameters defined at melting temperature, whose choice is a usually well-known difficulty when dealing with simplified thermal models. But in any case, these two approaches seem quite satisfactory; the linear scaling of $R^{-1}$ with the welding speed is well verified and should be useful for different purposes such as the preparation or the analysis of experiments, or its use for some process control scheme. 
${ }^{1} \mathrm{D}$. Rosenthal, "The theory of moving sources of heat and its application to metal treatments," Trans. ASME 68, 849-866 (1946).

${ }^{2}$ D. T. Swift-Hook and A. E. F. Gick, "Penetration welding with lasers," Weld. Res. Suppl. J. 52, 492-499 (1973).

${ }^{3}$ J. Dowden, N. Postacioglu, M. Davis, and P. Kapadia, "A keyhole model in penetration welding with a laser," J. Phys. D Appl. Phys. 20, 36-44 (1987).

${ }^{4} \mathrm{~F}$. Noller, in Proceedings of 3rd CISFFEL, International Colloquium on Welding and Melting by Electrons and Laser Beam (Lyon, France, 1983), pp. 89-97.

5 J. Kroos, U. Gratzke, and G. Simon, "Towards a-self-consistent model of the keyhole in penetration laser beam welding," J. Phys. D Appl. Phys. 26, 474-480 (1993).

${ }^{6}$ K. N. Lankalapalli, J. F. Tu, and M. Gartner, "A model for estimating penetration depth of laser welding processes," J. Phys. D Appl. Phys. 29, 1831-1841 (1996).

${ }^{7}$ A. Kaplan, "A model of deep penetration laser welding based on calculation of the keyhole profile," J. Phys. D Appl. Phys. 27, 1805-1814 (1994).

${ }^{8}$ D. Sudnik, D. Radaj, and W. Erofeew, "Computerized simulation of laser beam welding, modelling and verification,” J. Phys. D Appl. Phys. 29, 2811-2817 (1996).

${ }^{9} \mathrm{H}$. Ki, P. S. Mohanty, and J. Mazumder, "Modeling of laser keyhole welding: Part I. Mathematical modeling, numerical methodology, role of recoil pressure, multiple reflections, and free surface evolution," Metall. Mater. Trans. A 33, 1817-1830 (2002).

${ }^{10}$ M. Geiger, K. H. Leitz, H. Koch, and A. Otto, “A 3D transient model of keyhole and melt pool dynamics in laser beam welding applied to the joining of zinc coated sheets," Prod. Eng. 3, 127-36 (2009).

${ }^{11}$ S. Pang, L. Chen, J. Zhou, Y. Yin, and T. Chen, "A three-dimensional sharp interface model for self-consistent keyhole and weld pool dynamics in deep penetration laser welding,” J. Phys. D Appl. Phys. 44, 025301 (2011).

${ }^{12}$ M. Courtois, M. Carin, P. Masson, S. Gaied, and M. Balabane, "A new approach to compute multi-reflections of laser beam in a keyhole for heat transfer and fluid flow modelling in laser welding," J. Phys. D Appl. Phys. 46, 505305 (2013).

${ }^{13}$ W. Tan and Y. C. Shin, "Analysis of multi-phase interaction and its effects on keyhole dynamics with a multi-physics numerical model," J. Phys. D: Appl. Phys. 47, 345501 (2014).

${ }^{14}$ H. Zhao, W. Niu, B. Zhang, Y. Lei, M. Kodoma, and T. Ishide, "Modelling of keyhole dynamics and porosity formation considering the adaptive keyhole shape and three-phase coupling during deep-penetration laser welding," J. Phys. D: Appl. Phys. 40, 5753-66 (2011).

${ }^{15}$ M. Schneider, L. Berthe, R. Fabbro, and M. Muller, "Measurement of laser absorptivity for operating parameters characteristic of laser drilling regime," J. Phys. D: Appl. Phys. 41, 155502 (2008).

${ }^{16}$ R. Fabbro, "Melt pool and keyhole behavior analysis for deep penetration laser welding," J. Phys. D Appl. Phys. 43, 445501 (2010).

${ }^{17} \mathrm{~V}$. Semak and A. Matsunawa, "The role of recoil pressure in energy balance during laser materials processing," J. Phys. D Appl. Phys. 30, 2541-2552 (1997)

${ }^{18}$ W. Schulzt, D. Beckert, J. Franket, A. Kemmerlingt, and G. Herzigert, "Heat conduction losses in laser cutting of metals," J. Phys. D Appl. Phys. 26, 1357-1363 (1993).

${ }^{19}$ H. S. Carslaw and J. C. Jaeger, Conduction of Heat in Solids, 2nd ed. (Clarendon, Oxford, 1962).

${ }^{20} \mathrm{~A}$. Gouffe, "Correction d'ouverture des corps noirs artificiels compte tenu des diffusions multiples internes," Revue d'Optique 24, 1-10 (1945).

${ }^{21}$ M. Beck, "Modellierung des Lasertiefschweissens," Ph.D. Thesis, University of Stuttgart, Germany, 1996.

${ }^{22}$ R. Fabbro, K. Hirano, and S. Pang, "Analysis of the physical processes occurring during deep penetration laser welding under reduced pressure," J. Laser App. 28, 022427 (2016).

${ }^{23}$ See https://www.comsol.com

${ }^{24}$ W. J. Suder and S. Williams, "Power factor model for selection of welding parameters for CW laser welding," Opt. Laser Technol. 56, 223-229 (2014).
${ }^{25}$ A. Heider, P. Stritt, R. Weber, and T. Graf, in Proceedings of ICALEO Conference (San Diego, USA, 2014), pp. 343-348.

${ }^{26}$ K. C. Mills, Recommended Values of Thermophysical Properties for Selected Commercial Alloys (Woodhead Publishing Limited, Abington Cambridge, England, 2002).

\section{Meet the authors}

Remy Fabbro is presently Emeritus Research Director at CNRS. His scientific career has always been devoted with laser-matter interaction, and since 1989, he is involved in the study of fundamental processes about industrial lasers applications, concerning laser welding and cutting processes (laser interaction, hydrodynamics, plasma, modeling, ...), and laser-shock processing (modeling of confined interaction mode, shock wave characterization, surface treatment). He published more than 120 papers in international journals and about 200 conference proceedings.

Morgan Dal is Associate Professor at PIMM laboratory at the Arts et Métiers Engineering School. Its research fields are mainly devoted to the numerical simulation of different laser applications, such as laser welding, additive manufacturing, or surface treatment. Since few years, in order to improve simulation efficiency, he is also studying the measurement of thermal properties of metals with the help of laser.

Patrice Peyre is a Research Director at CNRS. He has been working on materials transformation with lasers for 25 years. He has published more than 60 scientific papers on various topics (laser-shock peening, dissimilar welding, additive manufacturing). He is currently in charge of additive layer additive manufacturing activities in PIMM laboratory.

Frédéric Coste is working on laser applications since 1990, mainly on high thickness welding of carbon and stainless steel, laser-matter interaction instrumentation and laser surface treatment. Since 2009, he is in charge of the laser workshop at PIMM laboratory, in the Arts et Métiers ParisTech school. His main interest is now on the study of metallic pool under neutral and reactive gazes, and additive manufacturing.

Matthieu Schneider received his $\mathrm{PhD}$ in 2006. He has over 30 publications in peer-reviewed journals and conference proceedings and two patents related to laser processing and photonic science. He is an Associate Professor at the Arts et Métiers Engineering School and a member of the LIA. He is in charge of the Mechanical Engineering Semestre. His Research deals with laser processing (cutting, welding, drilling, and additive manufacturing).

Valerie Gunenthiram is a PhD student at the PIMM laboratory (Arts et Métiers ParisTech Engineering School, Paris), is currently studying the various phenomena that occur in selective laser melting, more specifically the laserpowder-melt pool interaction and related processes controlling the hydrodynamics of these melt pools and their consequences on the porosity origin. 